\title{
Comparing the effects of different amounts of fluid treatments in addition to analgesia in patients admitted to the emergency department with renal colic: A randomized study
}

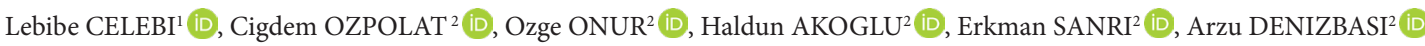 \\ ${ }^{1}$ Department of Emergency Medicine, Nyala Sudan, Turkish Research and Training Hospital, Nyala, Sudan. \\ ${ }^{2}$ Department of Emergency Medicine, School of Medicine, Marmara University, Pendik, Istanbul, Turkey.
}

Corresponding Author: Ozge ONUR

E-mail: ozberkozge@gmail.com

Submitted: 27.07.2021 Accepted: 14.10.2021

\begin{abstract}
Objective: There are a limited number of studies examining the effect of fluid administration for acute pain relief in patients with renal colic. We aim to evaluate whether intravenous fluid of different amounts will make a difference regarding pain, in patients who presented to the emergency department (ED) with flank pain.

Patients and Methods: This single-center, prospective, randomized clinical trial was performed at the ED of a university hospital. Subjects were randomly assigned to three groups. All received an intramuscular (IM) injection of $75 \mathrm{mg}$ diclofenac sodium and $3 \mathrm{mg}$ intravenous (IV) morphine. While group 1 did not receive extra treatment, group 2 received $100 \mathrm{cc} / \mathrm{hr}$ physiological serum (PS), and group 3 received $500 \mathrm{cc} / \mathrm{hr}$ PS. Pain was assessed by using the visual analogue scale (VAS) ruler for 6 hours.

Results: A total of 201 patients were included. Mean age was $36.16 \pm 9.85$. At 60 min mean VAS scores were $3.55 \pm 1.24$ in the first group, $4.42 \pm 1.87$ in the second group and $5.02 \pm 1.92$ in the third group. In the group fluid not given, pain decrease was faster than others. At 240 min mean VAS scores were similar in all groups.

Conclusion: This study indicates that IV fluids given to patients with renal colic pain was not effective in pain relief.

Keywords: Renal colic, Analgesia, Intravenous fluid, Urolithiasis, VAS
\end{abstract}

\section{INTRODUCTION}

Urinary system stone complaints appear as a common disease with a high incidence in developed countries. 8-15\% of Europeans and North Americans have urolithiasis [1]. In recent years, an increase in the incidence of urinary tract stones has been detected in developed countries [2,3]. Since, symptoms of anorexia, nausea and vomiting are common, fluid supplementation, maintenance of IV physiological serum (PS) infusion therapy is generally administered to prevent dehydration and fluid loss in patients with renal colic. However, it is not known exactly how much fluid should be given to the patients, and what the effects are of the fluids administered for pain and stone passage.

There are a limited number of studies examining the effect of fluid administration on acute pain relief. In literature, there are only two prospective and randomized studies, which found that a high amount of fluid and diuretic therapy accelerate stone passage in cases of acute renal colic $[4,5]$.
Our primary aim is to investigate whether intravenous fluid therapy has a role in reducing acute pain in the first 6 hours of admission to the emergency department (ED). We also aimed to analyze the general demographic data, comorbidities, family history and daily water consumption of patients who were admitted to the ED with renal colic.

\section{PATIENTS and METHODS}

\section{Study Design}

This study was designed as a prospective, randomized, doubleblind, double-dummy, placebo-controlled, single-dose, singlecenter study. Marmara University, School of Medicine, Ethics Committee approved the study (09.2019.870). CONSORT guidelines were followed. We adhered to the principles of the Declaration of Helsinki. Written informed consent was obtained.

How to cite this article: Celebi L, Ozpolat C, Onur O, Akoglu H, Sanri E, Denizbasi A. Comparing the effects of different amounts of fluid treatments in addition to analgesia in patients admitted to the emergency department with renal colic: A randomized study. Marmara Med J 2022: 35(1):17-22. doi: 10.5472/marumj.1061180 
Our study was conducted on patients who were admitted to Marmara University Pendik Training and Research Hospital, Istanbul. The population of the study consists of all patients who applied to ED with complaints of flank pain. All patients who admitted to ED with acute flank pain, located in the right or left flank under the ribs extending from the axillary line to the hip and which can be described as pain in the groin, were examined by ED physicians. Patients who were suspected as suffering from renal colic during their examinations, underwent abdominal tomography for diagnosis and differential diagnosis. All patients who were found to have opacities were diagnosed as having stones in the urinary tract and were included in the study.

Patients under the age of 18, pregnant women, those with the Glasgow Coma Scale (GCS) $<15$ and those who showed limited cooperation, had had no oral intake for 12 hours, had fever $>$ $38.3^{\circ} \mathrm{C}$ and systolic blood pressure $<90 \mathrm{mmHg}$, who had used analgesics or antipyretics for any reason in the last 2 days, who were diagnosed with kidney failure, congestive heart failure, or pyelonephritis detected at the stage of diagnosis, who had undergone urinary tract surgery, who had a known allergy to any of the study drugs; who gave incomplete information or voluntarily left the ED during the observation period, and those who did not consent to participate in the study were excluded.

Patients included in the study were randomized by the closed envelope method. The treatment of each patient admitted to the observation room was determined in line with the group to which the patient was randomized and the treatment was initiated by the nurse on duty. Randomization was done using the Randomizer.org program [6].

\section{Procedures for Treatment of Groups}

Patients were randomized as Group 1,2 and 3. All groups were administered intravenously $75 \mathrm{mg}$ diclofenac sodium as a nonsteroidal anti-inflammatory drug and $3 \mathrm{mg}$ morphine as a narcotic analgesic at time T0. In the first group, fluid was not given to the patients in addition to analgesic, but a nurse closed the serum set dosiflow with a cardboard and administered it to the patients. The patients were informed that fluid therapy was performed using serum. Unlike the first group, Group 2 had $100 \mathrm{cc} /$ hour $0.9 \% \mathrm{NaCl}$ solution as an infusion for 6 hours. Since, the intravenous fluid infusion given was sealed with a cardboard, the patients were prevented from seeing the fluid ingested. Unlike the other groups, Group 3 had $500 \mathrm{cc} /$ hour $0.9 \% \mathrm{NaCl}$ solution given as an infusion for 6 hours. Also the intravenous fluid infusion given was covered with a cardboard, and the patient was prevented from seeing the fluid ingested. Appropriate dose of morphine IV (not exceeding $10 \mathrm{mg}$ in total) was administered as rescue therapy, if necessary, to patients whose pain was not relieved and who still needed analgesics in all groups.

Analgesic administration time was determined as T0, and the serum for the patients was started at the same time.

Hemogram, kidney function tests and complete urinalysis were sent from the patients determined as study candidates.
Written informed consent was obtained from patients who were found to have urinary tract stones on abdominal tomography and their treatment was initiated. After pain relief treatment of the patients was started, a questionnaire was filled in by a research assistant.

Patients included in the study were followed up in the ED for 6 hours. During the follow-up, any complications or drug side effects were noted. The patients who completed the study were started on home analgesic treatment and were referred to the outpatient follow-up or to the ward, according to the guidelines. Physicians who were blind to the study groups asked the patients about pain by visual analogue scale (VAS) which is a validated, subjective measure for acute and chronic pain. Scores were recorded by making a handwritten mark on a $10-\mathrm{cm}$ line representing a continuum between "no pain" and "acute pain" charts at certain intervals starting from the treatment, at 0,15 , $45,60,90,120,150,180$ and $240 \mathrm{~min}$.

Total research population was 272. Sixty-two patients were excluded from the study ( 39 patients because they had been previously operated for urinary tract stones, 9 patients were diagnosed as having pyelonephritis, and 14 patients did not consent to the study). 210 patients were randomized. After randomization, 9 patients could not be included in the analysis due to missing data, so the data of 201 patients were analyzed. Flow chart is as Figure 1.

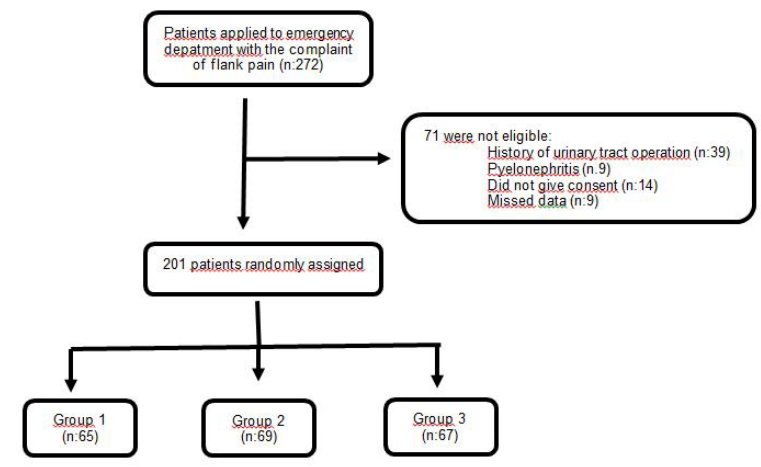

Figure 1. Study flow chart

\section{Calculating the Sample Size}

The sample size was calculated as 58 patients for each group in which the VAS score difference of at least $1 \mathrm{~cm}$ between at least two of the groups at any time could be detected, as the within-group standard deviation is $2 \mathrm{~cm}$, but the type 1 error is $5 \%$ and the power is $80 \%$. It was decided to recruit $20 \%$ more patients for each group, and at the end of the study, the groups were completed as 67, 69 and 65 patients. After the study was concluded, the power to detect the difference between the 3 averages of the study at 1 hour of treatment was determined to be $99 \%$. In the whole study, type 1 error was accepted as $5 \%$. 


\section{Statistical Analysis}

Continuous variables are given their mean and standard deviations and 95\% confidence intervals, and the frequencies and percentages of categorical variables are given. Comparisons of continuous variables between groups were made with analysis of variance, and comparisons of categorical variables between groups were made with Fischer's exact test. The difference between the percentages of the categorical variables between the groups and the confidence intervals of this difference were also calculated and the effects were reported. The variation between consecutive measurements of VAS scores and whether there is a difference between these changes between groups was evaluated by analysis of the variance of consecutive measurements. P-value less than 0.001 was evaluated as statistically significant.

Before the study, the online sample size calculation program StatsToDo was used for sample size and post-hoc power calculation (https://www.statstodo.com/SSizAOV_Pgm.php).

All other analyses and graph breakdowns were calculated using Medcalc version 15.11.4 (Medcalc, Belgium).

\section{RESULTS}

Mean age of all 201 patients was $36.16 \pm 9.85$ (95\% GA 34.79 - 37.53) and $171(85.10 \%)$ of those were male. Among those who enrolled in the study, $60(29.90 \%)$ patients had a history of nephrolithiasis, while $64(31.80 \%)$ had a positive family history for it. Considering the water consumption of patients, $51(25.40 \%)$ of them drank 5 to 6 glasses of water, $97(48.30 \%)$ drank 7 to 10 glasses and 53 (26.40\%) drank over 10 glasses a day. As shown in Table I, the basic demographics and past medical and family histories of patients were similar in each group except for those with a history of diabetes.

Table I. Distribution of patients according to demographics and comorbid diseases

\begin{tabular}{|c|c|c|c|c|c|}
\hline \multicolumn{2}{|c|}{ Demographics } & $\begin{array}{c}\text { Group 1 } \\
(\mathrm{n}=67)\end{array}$ & $\begin{array}{c}\text { Group } 2 \\
(\mathrm{n}=69)\end{array}$ & $\begin{array}{c}\text { Group } 3 \\
(\mathrm{n}=65)\end{array}$ & $\mathbf{p}$ \\
\hline \multicolumn{2}{|c|}{$\begin{array}{l}\text { Age (year) } \\
\text { mean } \pm \text { SD }(95 \% \mathrm{CI})\end{array}$} & $\begin{array}{c}37.20 \pm 10.60 \\
(34.60- \\
39.80)\end{array}$ & $\begin{array}{c}36.30 \pm 10.40 \\
(33.80- \\
38.80)\end{array}$ & $\begin{array}{c}35.00 \pm 8.40 \\
(32.90- \\
37.00)\end{array}$ & 0.42 \\
\hline \multicolumn{2}{|l|}{ Sex (male) n(\%) } & $61(91.00)$ & $55(79.70)$ & $55(84.60)$ & 0.17 \\
\hline \multicolumn{2}{|c|}{$\begin{array}{l}\text { History of } \\
\text { nephrolithiasis }(+) \text { n (\%) }\end{array}$} & $22(32.80)$ & $21(30.40)$ & $17(26.20)$ & 0.69 \\
\hline \multirow{3}{*}{$\begin{array}{l}\text { Water } \\
\text { consumption } \\
\text { (glass/day) n (\%) }\end{array}$} & $5-6$ & $18(26.90)$ & $14(20.30)$ & $19(29.20)$ & \multirow[t]{3}{*}{0.62} \\
\hline & 7-10 & $29(43.30)$ & $36(52.20)$ & $32(49.20)$ & \\
\hline & $>10$ & $20(29.90)$ & $19(27.50)$ & $14(21.50)$ & \\
\hline \multicolumn{2}{|c|}{$\begin{array}{l}\text { Family history for } \\
\text { nephrolithiasis (+) n (\%) }\end{array}$} & $17(25.40)$ & $24(34.80)$ & $23(35.40)$ & 0.37 \\
\hline \multicolumn{2}{|c|}{ History of DM n (\%) } & $2(3.00)$ & $7(10.10)$ & $1(1.50)$ & 0.04 \\
\hline \multicolumn{2}{|c|}{ History of HT n (\%) } & $7(10.40)$ & $9(13.00)$ & $2(3.10)$ & 0.11 \\
\hline \multicolumn{2}{|c|}{ History of CAD n (\%) } & $3(4.50)$ & $4(5.80)$ & $1(1.50)$ & 0.43 \\
\hline
\end{tabular}

$D M$ : diabetes mellitus, HT: hypertention, CAD: coronary artery disease, $S D$ : standard deviation, CI: confidence interval. $p<0.05$ is statistically significant.
According to the computed tomography (CT) findings in the patients, concurrent hydronephrosis was detected in 177 $(77.10 \%)$ and mean diameter of calculus was $5.83 \pm 3.79 \mathrm{~mm}$ (95\% GA 5.30 - 6.36). Calculi were located in the renal pelvis in $45(22.40 \%)$ patients, in the ureters in $105(52.20 \%)$ patients, in the ureterovesical junction in $34(16.90 \%)$ patients and in the bladder in $17(8.50 \%)$ patients. There was no statistically significant difference between groups regarding the presence of hydronephrosis, size and location of calculi (Table II).

Table II. Distribution of patients according to CT findings

\begin{tabular}{|c|c|c|c|c|c|}
\hline \multicolumn{2}{|l|}{ CT finding } & $\begin{array}{c}\text { Group 1 } \\
(n=67)\end{array}$ & $\begin{array}{c}\text { Group 2 } \\
(\mathrm{n}=69)\end{array}$ & $\begin{array}{c}\text { Group } 3 \\
(n=65)\end{array}$ & p \\
\hline \multicolumn{2}{|c|}{$\begin{array}{l}\text { Diameter of calculus (mm) } \\
\text { mean } \pm \text { SD }(95 \% \text { CI })\end{array}$} & $\begin{array}{c}5.90 \pm 2.90 \\
(5.20-6.60)\end{array}$ & $\begin{array}{c}6.30 \pm 4.40 \\
(5.20-7.40)\end{array}$ & $\begin{array}{c}5.30 \pm 3.90 \\
(4.30-6.30)\end{array}$ & 0.32 \\
\hline \multicolumn{2}{|c|}{ Hydronephrosis n (\%) } & $50(74.60)$ & $51(73.90)$ & $54(83.10)$ & 0.38 \\
\hline \multirow{4}{*}{$\begin{array}{l}\text { Location } \\
\text { of calculus } \\
\mathrm{n}(\%)\end{array}$} & Renal pelvis & $16(23.90)$ & $18(26.10)$ & $11(16.90)$ & 0.80 \\
\hline & Ureters & $32(47.80)$ & $37(53.60)$ & $36(55.40)$ & \\
\hline & Ureterovesical & $12(17.90)$ & $10(14.50)$ & $12(18.50)$ & \\
\hline & $\begin{array}{l}\text { junction } \\
\text { Bladder }\end{array}$ & $7(10.40)$ & $4(5.80)$ & $6(9.20)$ & \\
\hline
\end{tabular}

SD: standard deviation, CI: confidence interval. $p<0.05$ is statistically significant.

Intravenous morphine treatment was applied as rescue treatment to 18 of $67(26.90 \%)$ patients in Group 1, 38 of $69(55.10 \%)$ patients in Group 2 and 51 of $65(78.50 \%)$ patients in Group 3. More rescue treatment was needed in Group 3, who were given the most vigorous fluid resuscitation, and the least in Group 1 who were not given any IV fluid. The difference between groups was statistically significant $(\mathrm{p}<0.01)$.

Table III. Comparison of VAS scores in groups

\begin{tabular}{|c|c|c|c|c|}
\hline \multirow{2}{*}{ Time } & \multicolumn{2}{|c|}{ VAS score (mm) mean \pm SD $(\mathbf{9 5} \% \mathbf{C I})$} & \multirow{2}{*}{ p } \\
\cline { 2 - 5 } & Group 1 (n=67) & $\begin{array}{c}\text { Group 2 } \\
(\mathbf{n}=\mathbf{6 9})\end{array}$ & $\begin{array}{c}\text { Group 3 } \\
(\mathbf{n}=\mathbf{6 5})\end{array}$ & \\
\hline $\min 0$ & $7.73 \pm 0.65$ & $7.77 \pm 0.67$ & $7.97 \pm 0.73$ & 0.099 \\
& $(7.56-7.89)$ & $(7.61-7.93)$ & $(7.80-8.14)$ & \\
\hline $\min 15$ & $6.24 \pm 1.15$ & $6.41 \pm 1.20$ & $6.63 \pm 1.18$ & 0.183 \\
& $(5.96-6.53)$ & $(6.12-6.87)$ & $(6.34-6.92)$ & \\
\hline $\min 45$ & $5.11 \pm 1.66$ & $5.71 \pm 1.64$ & $5.80 \pm 1.50$ & 0.012 \\
& $(4.75-5.46)$ & $(5.37-6.06)$ & $(5.45-6.16)$ & \\
\hline $\min 60$ & $3.55 \pm 1.24$ & $4.42 \pm 1.87$ & $5.02 \pm 1.92$ & $<\mathbf{0 . 0 0 1}$ \\
& $(3.13-3.96)$ & $(4.02-4.83)$ & $(4.60-5.43)$ & \\
\hline $\min 90$ & $2.27 \pm 1.35$ & $3.23 \pm 1.94$ & $3.69 \pm 1.80$ & $<\mathbf{0 . 0 0 1}$ \\
& $(1.86-2.69)$ & $(2.82-3.64)$ & $(3.27-4.11)$ & \\
\hline $\min 120$ & $1.23 \pm 1.15$ & $2.09 \pm 1.66$ & $2.45 \pm 1.78$ & $<\mathbf{0 . 0 0 1}$ \\
& $(0.85-1.60)$ & $(1.72-2.46)$ & $(2.07-2.83)$ & \\
\hline $\min 150$ & $0.58 \pm 0.84$ & $1.07 \pm 1.23$ & $1.29 \pm 1.27$ & 0.010 \\
& $(0.30-0.85)$ & $(0.80-1.34)$ & $(1.02-1.57)$ & \\
\hline $\min 180$ & $0.24 \pm 0.47$ & $0.45 \pm 0.72$ & $0.58 \pm 0.83$ & 0.015 \\
& $(0.08-0.41)$ & $(0.29-0.61)$ & $(0.42-0.75)$ & \\
\hline $\min 240$ & $0.12 \pm 0.33$ & $0.10 \pm 0.30$ & $0.22 \pm 0.45$ & 0.157 \\
& $(0.03-0.21)$ & $(0.02-0.19)$ & $(0.13-0.31)$ & \\
\hline
\end{tabular}

Min: minute, SD: standard deviation, CI: confidence interval. $p<0.05$ is statistically significant. 
In time, pain relief was seen in all groups. Considering mean VAS scores, there was no statistically significant difference between groups at time zero, 15 and $45 \mathrm{~min}$. On the other hand, Group 1 had statististically significant lower mean VAS score at 60, 90 and $120 \mathrm{~min}$. Statistically significant differences in VAS scores were not observed between groups either at 150, 180 or 240 minutes (Table III).

The decrease in consecutive VAS scores was significantly different both in groups and between groups $(\mathrm{p}<0.001 ; \mathrm{p}<0.001$ respectively).

Rescue treatment was not needed in a total of 93 (46 of 67 (73.10\%) patients in Group 1, 31 of 69 (44.90\%) patients in Group 2 and 14 of 65 (21.50\%) patients in Group 3) patients. There was no statistically significant difference in mean VAS scores between groups $(\mathrm{p}=0.21)$.

None of the patients were discharged prior to 6 hours.

\section{DISCUSSION}

Renal colic is a common diagnosis in ED. In this study, we investigated the effect of the amount of fluid administered as acute pain treatment, using different amounts of intravenous fluid in addition to analgesic treatment for patients who were admitted to ED with flank pain and detected urinary system stones on their CT images. As a result of the study, we found that traditionally administered intravenous fluid did not help, and even the group receiving fluid needed more analgesic treatment.

The mean age of the patients included in the study was found to be $36.16 \pm 9.85$. In a study conducted in France and Germany, the most common age range for urinary stones was determined as 40-49 in men and 30-39 in women [7]. The average age value found in our study is compatible with the literature.

Of the patients in our study, $85.10 \%$ were men and the prevalence rate of kidney stones was higher than in many studies in favor of men. In many studies published abroad, it has been reported that there has been an increase in urinary system stone disease in women in recent years, and the rate of nephrolithiasis in men and women has decreased from 3: 1 to 1.30: 1 [8-11].

Almost half of the patients in our study stated that they consumed 1.5-2 liters of water a day, 25\% consumed less than this. In a study reporting that when 2.5 liters of urine output is achieved due to a daily intake of more than 2 liters of water, it reduces the recurrence of kidney stones [12]. In our study, $75 \%$ of the patients consumed less than 2 liters of water.

Family history was positive in $31.80 \%$ patients. Most frequently, $47.60 \%$ patients had relatives other than mother, father and sibling; Subsequently, $31.70 \%$ patients had a history of stones in the father and $7.90 \%$ patients in the sibling. Although, there is a familial predisposition in urinary system stone disease, the relevant genes are not yet known [13].

Stone size in the CT images was evaluated and the average stone diameter was found to be $5.83 \pm 3.79$. In the study conducted by Edna et al., in Norway, the average stone size was found to be 4 $\mathrm{mm}$, and $3.40 \mathrm{~mm}$ in the study of Springhart et al. $[4,14]$.
In our study, the most common area where stones were detected was the ureter. Stones were detected in the ureteropelvic junction (UPJ) in $22.40 \%$ patients, in the ureterovesical junction (UVJ) in $16.90 \%$ patients, and in the bladder in $8.50 \%$ patients. In a study, the most common place where the stones were found was the UVJ and the proximal ureter [15]. In our study, the results were similar due to anatomical reasons.

When hydronephrosis occurs due to an acute cause, it does not remain asymptomatic as it is when due to a chronic cause, but creates colic-style pain. In various studies, the rate of hydronephrosis in urinary system stones has been reported as $69-83 \%[16,17]$. The rates of patients with hydronephrosis in our study are similar to those stated in the sources. Based on this data, it can be assumed that most of the patients included in our study had obstruction.

Due to the intense pain in renal colic, analgesics are used in the treatment. General practice in EDs is to give IV therapy in addition to analgesic therapy. Theoretically, it is thought that the IV fluid given will increase the hydrostatic pressure in the ureter, allowing the stone to move rapidly in the ureter, and the patient's pain will be relieved faster as a result of the quicker reduction of the stone. However, there are no clear recommendations in the guidelines regarding the effect of fluid administration on pain in patients and how much fluid should be given to patients. The number of studies conducted to investigate the effect of fluid treatments administered for acute pain attacks caused by kidney stones is limited. There are two prominent prospective and randomized studies on this subject. The first study highlighted in the Cochrane database is the work of Edna et al., in 1983 [4]. In this study, 60 patients were randomized to groups $A$ and $B$; liquid was not given to group A for 6 hours, and 3 liters fluid infusion was given to group B. Pethidine was used as an analgesic. There was no difference between the groups in terms of pain and surgical intervention rates at the end of 6 hours, which were the endpoints. As a result of this study, it was found that IV fluid was not beneficial in renal colic pain. The second randomized controlled study emphasized in the Cochrane database was conducted in 2006 by Springhart et al., to investigate the effect of IV fluid administered in acute pain treatment of renal colic, 43 patients were enrolled in the study; patients in the first group, received 4 lt in 4 hours normal saline infusion, and those in the second group received $20 \mathrm{cc} /$ hour saline infusion [15]. Ketorolac and morphine were administered as an analgesic. In this study, no significant difference was found between the groups in terms of stone passage, additional medication need and hourly pain. The regression in the pain levels of the patients was similar in the groups with and without fluid. The results of these limited number of studies investigating the effect of IV fluid on pain in renal colic pain were in line with the results of our study. Also we found that even the group receiving fluid needed more analgesic treatment.

In our study, a faster pain response was detected in the mean VAS value of the first group, the group in which we did not give fluid, starting at $60 \mathrm{~min}$ and continuing at 90 and $120 \mathrm{~min}$ compared to the $2^{\text {nd }}$ and $3^{\text {rd }}$ groups in which we gave fluid. The group in which VAS averages were the highest during these time 
intervals, that is, the group in which the pain persisted, was the third group that received the most fluid. At 240 min mean VAS scores were similar between groups.

The reason for the delayed analgesic responses of the patients taking fluid may be that the administered fluid increased the volume of distribution by causing dilution in the applied analgesic doses. The significant difference between the VAS average values of the groups started to decrease from the $150^{\text {th }}$ min and the difference disappeared at the $240^{\text {th }} \mathrm{min}$, which was the ending point. At the end of the follow-up period, the level of pain in the groups with and without intravenous fluids questioned the efficacy of the fluid therapy routinely applied to most renal colic patients in many centers.

Considering the analgesics administered, morphine, which was given as rescue therapy to patients whose pain relief could not be obtained sufficiently, creates a significant statistical difference between the groups. The fact that the least need for rescue therapy was in Group 1 with $26.90 \%$ patients, who were also not given fluids, shows that fluid therapy increases the need for additional analgesics. One of the reasons why the number of patients needing additional doses of analgesics is clearly higher in the groups that are given fluid may be that the increased intravascular volume causes increased urine flow rate, a dramatic increase in ureteral peristalsis, which may possibly cause stone pushing. In this system, which has been obstructed due to the stone, the increase in pressure and tension caused by the IV fluid may cause an increase in the patient's pain. Another reason may be the accumulation of urine at a high urine flow rate and the fact that urine transport occurs according to the pressure gradient in the open tube $[18,19]$.

As a result of our study, it was found that pain in renal colic patients with and without fluid therapy regressed to the same level at the end of the follow-up of the patients, and the need for additional medication in the group with fluid was higher than in the group without fluid. Further large-scale studies in which the patients enrolled in the study are evaluated in more detail are required to determine the effect and benefit on their functions,

There are limitations to this study. Patients admitted to ED with flank pain and who were diagnosed with urolithiasis as a result of their examinations were included in our study. This does not mirror the general population. Also, in our study, patients whose underlying heart failure would pose a risk to fluid administration were excluded Therefore, the data of these patients are lacking.

Before starting the study, although all physicians working in the ED received training for the research, and the differential diagnosis and diagnosis algorithm were explained, the fact that different physicians examined the patients may have created a bias in patient selection. Since, our study was conducted within the boundaries of the ED, some data may be said to be missing. We conclude that IV fluids given to patients with renal colic pain was not effective in pain relief.

\section{Compliance with Ethical Standards}

Ethical Approval: This study was approved by the Ethics Committee of Marmara University, School of Medicine
(09.2019.870). We adhered to the principles of the Declaration of Helsinki. Written informed consent was obtained.

Financial Support: No specific funding was received.

Conflict of Interest: There are no conflicting interests.

Author contributions: LC, CO, OO and ES: Conceived the study and designed the trial, LC, CO, HA and AD: Supervised the conduct of the trial and data collection, LC: Undertook recruitment of participating patients and managed the data, including quality control, CO, ES and HA: Provided statistical advice on the study design and analyzed the data, OO: Chaired the data oversight committee, LC, CO, OO,ES, AD and HA: Drafted the article, and all authors contributed substantially to its revision, CO: Takes responsibility for the paper as a whole.

\section{REFERENCES}

[1] Dellabella M, Milanese G, Muzzonigro G. Randomized trial of the efficacy of tamsulosin, nifedipine and phloroglucinol in medical expulsive therapy for distal ureteral calculi. J Urol 2005;174:167-72. doi: 10.1097/01.ju.000.016.1600.54732.86

[2] Trinchieri A, Coppi F, Montanari E, Del Nero A, Zanetti G, Pisani E. Increase in the prevalence of symptomatic upper urinary tract stones during the last ten years. Eur Urol 2000;37:23-5. doi: 10.1159/000020094

[3] Hesse A, Brandle E, Wilbert D, Kohrmann KU, Alken P. Study on the prevalence and incidence of urolithiasis in Germany comparing the years 1979 vs. 2000. Eur Urol 2003;44:709-13. doi: 10.1016/s0302-2838(03)00415-9

[4] Edna TH, Hesselberg F. Acute ureteral colic and fluid intake. Scand J Urol Nephrol 1983;17:175-8. doi: 10.3109/003.655.98309180164

[5] Kirschner J, Wilbur L. Do fluids facilitate stone passage in acute ureteral colic? Ann Emerg Med 2013;62:36-7. doi: 10.1016/j.annemergmed.2012.08.004

[6] Urbaniak G C, Plous S. Research randomizer [Computer software]. Available at: http://www.randomizer.org/ Accessed on:04.01.2020

[7] Daudon M, Dore JC, Jungers P, Lacour B. Changes in stone composition according to age and gender of patients: a multivariate epidemiological approach. Urol Res 2004;32:2417. doi: 10.1007/s00240.004.0421-y.

[8] Schade GR, Faerber GJ. Urinary tract stones. Prim Care 2010;37:565-81. doi: 10.1016/j.pop.2010.05.003.

[9] Scales CD Jr, Curtis LH, Norris RD, et al. Changing gender prevalence of stone disease. J Urol 2007;177:979-82. doi: 10.1016/j.juro.2006.10.069.

[10] Romero V, Akpinar H, Assimos DG. Kidney stones: a global picture of prevalence, incidence, and associated risk factors. Rev Urol 2010;12:e86-96.

[11] Seitz C, Fajkovic H. Epidemiological gender-specific aspects in urolithiasis. World J Urol 2013;31:1087-92. doi: 10.1007/ s00345.013.1140-1.

[12] Fink HA, Akornor JW, Garimella PS, et al. Diet, fluid, or supplements for secondary prevention of nephrolithiasis: a 
systematic review and meta-analysis of randomized trials. Eur Urol 2009;56:72-80. doi: 10.1016/j.eururo.2009.03.031.

[13] Goldfarb DS, Fischer ME, Keich Y, Goldberg J. A twin study of genetic and dietary influences on nephrolithiasis: a report from the Vietnam Era Twin (VET) Registry. Kidney Int 2005;67:1053-61. doi: 10.1111/j.1523-1755.2005.00170.x.

[14] Springhart WP, Marguet CG, Sur RL, et al. Forced versus minimal intravenous hydration in the management of acute renal colic: a randomized trial. J Endourol 2006;20:713-6. doi: 10.1089/end.2006.20.713.

[15] Eisner BH, Reese A, Sheth S, Stoller ML. Ureteral stone location at emergency room presentation with colic. J Urol 2009;182:165-8. doi: 10.1016/j.juro.2009.02.131.
[16] Özbir S, Can O, Atalay HA, Canat HL, Çakır SS, Ötünçtemur A. Formula for predicting the impaction of ureteral stones. Urolithiasis 2020;48:353-60. doi: 10.1007/s00240.019.01152-y

[17] Kim SG, Jo IJ, Kim T, et al. Usefulness of protocolized pointof-care ultrasonography for patients with acute renal colic who visited emergency department: A randomized controlled study. Medicina (Kaunas) 2019; 55:717. doi: 10.3390/ medicina55110717.

[18] Wein AJM, Louis R. Kavoussi MD. Campbell-Walsh Urology. 11th Edition. Philadelphia: Elsevier, 2016:978-1003.

[19] Takaddus AT, Gautam P, Chandy AJ. A fluid-structure interaction (FSI)-based numerical investigation of peristalsis in an obstructed human ureter. Int J Numer Method Biomed Eng 2018;34:e3104. doi: 10.1002/cnm.3104 